\title{
CYTOLOGICAL EVALUATION OF NECROPSY GUIDED IMPRESSION SMEARS OF CHRONIC RESPIRATORY DISEASE OF CHICKENS
}

\author{
A. Khatun, J. A. Begum, F. Naznin, R. Parvin, M. M. Rahman, \\ S. M. Sayem ${ }^{2}$ and E. H. Chowdhury ${ }^{1}$ \\ Department of Pathology, Faculty of Veterinary Science, Bangladesh Agricultural University, \\ Mymensingh-2202, Bangladesh
}

\begin{abstract}
The study was conducted to develop a cytology based diagnostic tool for the diagnosis of avian Mycoplasma and E. coli infections at post mortem in the field condition. A total of 38 culture and PCR confirmed Mycoplasma, E. coli or mixed infected samples were used for this study. Lung impression smears were prepared on glass slide from the samples at post mortem examination. Inflammatory cells were counted on microscope after Giemsa staining. Cell counts were analyzed with Bonferroni joint confidence interval and Mann-Whitney $\mathrm{U}$ test. The average cell percentages in healthy cases were 73.54$81.66 \%, 9.63-13.37 \%$ and $7.42-14.38 \%$ for lymphocyte, heterophil, and macrophage, respectively. In case of Mycoplasma infection, average percentages of lymphocyte, heterophil and macrophages were $82.01-88.10 \%, 5.6$ to $8.16 \%$ and $4.52-9.68 \%$, respectively. In $E$. coli infection, average percentage of lymphocyte, heterophil and macrophages were found as $64.44-70.76 \%, 19.73-23.47 \%$ and $8.9-$ $12.7 \%$, respectively. In mixed infection, lymphocyte, heterophil and macrophage were found as $76.08-80.50 \%, 13.47-17.63 \%$ and 4.56 $-7.66 \%$, respectively. Statistical analyses revealed that in Mycoplasma infection number of lymphocyte and in E. coli infection number of heterophil increased significantly $(p<0.01)$. In $M C$ complex, number of heterophil increased and macrophages decreased significantly $(p<0.01)$. These findings could help identification of Mycoplasma, E. coli or Mycoplasma- E. coli complex at post mortem examination in the field condition.
\end{abstract}

Key words: CRD, mycoplasma, E. coli and cytology.

\footnotetext{
${ }^{1}$ Corresponding author email: emdad001@yahoo.com

${ }^{2}$ Department of Agricultural Statistics, Faculty of Agricultural Economics and Rural Sociology, Bangladesh Agricultural University, Mymensingh-2202, Bangladesh
}

Received: 07.05.2012 
A. Khatun et al.

\section{INTRODUCTION}

Poultry rearing is playing a vital role in the poverty alleviation and income generation in Bangladesh. Mycoplasmosis is an economically important disease of poultry caused by four commonly recognized pathogens - Mycoplasma gallisepticum, M. synoviae, M. meleagridis and M. iowae. M. gallisepticum and $M$. synoviae (Sikder et al., 2005) causing chronic respiratory disease (CRD) with significant mortality and decrease in egg production in chickens, turkeys and other avian species. In Bangladesh, the prevalence of Mycoplasmosis markedly increases in the winter season and may reach up to $61.45 \%$ which is a threat for poultry sector (Sikder et al., 2005). Very often it is confused with other respiratory diseases particularly with $E$. coli infection which sometimes make the treatment very expensive. Early diagnosis is the corner stone for the successful treatment, prevention and control strategies. The organism can be diagnosed by isolation, serological tests, culture and nucleic acid based tests (Nascimento et al., 2005). Conventional means of isolation or culture have proven to be tedious and time consuming and it requires special growth supplement, which make it an expensive option (OIE, 2008). It is important to choose a way for diagnosis that will be compatible with actual field condition, as well as facilities available. Clinical pathology has played a major role in the diagnosis of human and animal diseases. The importance and accuracy of cytological examination as a diagnostic tool has been studied and reviewed in the human and veterinary literature (Bottles et al., 1986, Cochand-Priollet et al., 1987, Campbell, 1988, Cowell et al., 1989) as an indirect method of diagnosis in case of infectious diseases which involve relatively non-invasive means of sampling from the specimens and could provide one step forward from post mortem diagnosis. This paper describes the use of cytological smear collected at necropsy for the diagnosis of avian Mycoplasma and E. coli infections which were compared with the histopathological changes.

\section{MATERIALS AND METHODS}

\section{Samples}

A total of 38 samples (Blood, lung exudates, swab from trachea and larynx) were collected from dead birds that were suspected to be the case of Mycoplasma or E. coli infections during November, 2008 to June, 2009. Ten (10) samples were collected from apparently healthy chickens at regular slaughter that assumed to be free from chronic respiratory diseases clinically as control. The samples that were confirmed as Mycoplasma or E. coli or mixed infection by culture and PCR were subjected for cytological evaluation.

\section{Pathological Studies}

Gross pathological examination was conducted on necropsy. Tissues from lungs, liver, trachea, heart and spleen organs were collected at necropsy and were preserved as per requirement. Part of the samples was fixed in $10 \%$ neutral buffered formalin for histopathological examination as per earlier described procedure (Luna 1968). 


\section{Cytology}

Clean glass slides were gently touched onto the lesion of lung and immediately lifted off and air dried, stained with Giemsa staining and observed under a microscope using a $100 \times$ magnification with oil immersion. The counting was done gradually from one side to another side and numbers of inflammatory cells were estimated by counting 300 cells. Only Mycoplasma, E. coli or complicated Mycoplasma infected cases determined on necropsy findings were considered under this count.

\section{Isolation of Mycoplasma and $E$. coli}

Lung and tracheal swabs were collected either in nutrient or Mycoplasma broth supplemented with supplement G (Oxoid, Milan, Italy) and kenamycin. The nutrient broth containing suspected swab was incubated in aerobic condition at $37^{\circ} \mathrm{C}$ for $48 \mathrm{~h}$. Upon growth of the organism, $100 \mu \mathrm{l}$ of inoculums (infected media) were inoculated into EMB agar plate (Oxoid, Milan, Italy). Plates were incubated at $37^{\circ} \mathrm{C}$ for $48 \mathrm{~h}$. After $48 \mathrm{~h}$, production of typical metallic sheen in EMB agar was considered as $E$. coli infection. Mycoplasma broth (Oxoid, Milan, Italy) containing suspected swab was incubated at $37^{\circ} \mathrm{C}$ for $72 \mathrm{~h}$. After growth, $100 \mu \mathrm{l}$ of Inoculum (infected media) were inoculated into Mycoplasma agar (Oxoid, Milan, Italy) with $20 \%$ of equine serum and plates were incubated for 10 days at $37^{\circ} \mathrm{C}$ in humid chamber with $10 \%$ $\mathrm{CO}_{2}$. After 2 or 3 passages Mycoplasma, when present, produced typical colony with fried egg appearance (Figure.1).

\section{Identification of isolated Mycoplasma using PCR}

Polymerase chain reaction (PCR) was used to detect the organisms following the procedure described by Lauerman et al., (1995). Commercial PCR kit (PCR Master Mixture Kit, GeNei ${ }^{\mathrm{TM}}$, Banglore Genei, BDA Industrial Suburb, Peenya, India) was used for this purpose. Mycoplasma gallisepticum vaccine; MG TS11(Merial Select, Inc, Gainesville, USA) was used as positive control for Mycoplasma gallisepticum during PCR. Wizard R Genomic DNA Purification Kit (Promega Corporation. 2800 Woods Hollow Road. Madison, USA) was used to extract the DNA from Mycoplasma vaccine and Mycoplasma isolates as per manufacturer's instructions. Primers and thermal profile were adopted from previous work (Lauerman et al., 1995). DNA amplification was performed in an oil-free thermal cycler (Master Cycler Gradient, Eppendorf, Germany). The amplified products were analyzed by $1.5 \%$ agarose gel (Promega Corp. Madison, WI, USA). The gel was visualized under UV light on a transilluminator (Labortechnik, Germany). The expected product size of M. gallisepticum and M. synoviae were of 900 and 497bp, respectively.

The diagnoses of the samples were made based on the following criteria (Table 1): 
Table 1: Criteria to identify Mycoplasma and E. coli infections

\begin{tabular}{ll}
\hline Diagnosis & Cultural characteristics/ PCR detection \\
\hline $\begin{array}{l}\text { Mycoplasma } \\
\text { infection }\end{array}$ & $\begin{array}{l}\text { Samples showed typical Mycoplasma colonies in Mycoplasma agar } \\
\text { and PCR positive }\end{array}$ \\
$\begin{array}{l}\text { E. coli infection } \\
\text { Samples showed positive growth with metallic sheen in EMB agar } \\
\text { and no colonies in Mycoplasma agar. }\end{array}$ \\
$\begin{array}{l}\text { E. coli complex } \\
\text { Samples showed positive growth with metallic sheen in EMB agar } \\
\text { and typical Mycoplasma colonies in Mycoplasma agar and PCR } \\
\text { positive. }\end{array}$ \\
\hline
\end{tabular}

\section{Statistical Analysis}

Cell counting results obtained from blood of Mycoplasma and E. coli infected birds and healthy control birds were analysed with Bonferroni joint confidence interval, Mann-Whitney U test (Johnson and Wichern, 2002) using the software MS Excel, SPSS and MINITAB 13.

\section{RESULTS AND DISCUSSION}

The samples that were confirmed as Mycoplasma or E. coli or mixed infection either by culture (Figure.1) or by PCR (Figure.2) were subjected for cytological evaluation. Pathological study was done to correlate the lesions with cytological smears. For normal healthy cases the average lymphocyte, heterophil, and macrophage counts were $73.54-81.66 \%, 9.63-13.37 \%$ and $7.42-14.38 \%$, respectively. Impression smears sampled from Mycoplasma infected birds showed predominant lymphocytic aggregates containing $82.01-88.10 \%$, on an average $8 \%$ increase when compared with normal healthy cases. The average cell percentage of heterophil and macrophage was found as 5.60-8.16\% and 4.52-9.68\% respectively, average 5\% decrease of both cells when compared to the normal healthy cases. On MannWhitney $U$ test it has been found that increase of lymphocyte and decrease of heterophil count significantly differ at $5 \%$ or $1 \%$ level of significance in comparison to normal healthy birds. These cytological changes closely correlated with the histopathological lesions of the same Mycoplasma confirmed birds where pneumonia with predominant lymphocytic infiltration was observed (Figure. 3). On the other hand, impression smears from E. coli infected birds showed an increasing trend of heterophil count (19.73-23.47\%), but lymphocyte and macrophage counts were relatively decreased (64.44-70.76\% and $8.9-12.7 \%)$. These findings also correspond to the findings of a previous histopathological study (Chandrashekhar, 2008). In same study statistical analysis showed decrease of lymphocytes and increase of heterophils and these significantly differ at $5 \%$ or $1 \%$ level of significance in comparison to normal healthy birds in E. coli infections. In case of mixed infection (Mycoplasma - 
E. coli complex), the average percent of lymphocyte was $76.08-80.58 \%$ in which the difference is insignificant in comparison to normal healthy cases. But, the percent of hetetrophils increased to $13.47-17.63 \%$ and the percent of macrophages relatively decreased to $4.56-7.66 \%$. In Mycoplasma -E. coli complex, the increase of lymphocytes is insignificant whereas significant difference existed between normal and Mycoplasma - E. coli complex for increased neutrophils and decreased macrophages. The relative numbers of different inflammatory cell types and their average percentage for normal healthy cases, Mycoplasma, E. coli infection and mixed infection are shown in tables $2-6$. Figure 4 shows predominant lymphocytic infiltration in a lung impression smear of Mycoplasma infected birds.

Predominance of mononuclear inflammatory cells (lymphocytes and macrophages) and a smaller number of heterophil indicates a chronic inflammatory process. Since Mycoplasmosis is a chronic respiratory disease, increased number of lymphocyte has been found in this study. Increased heterophil indicates acute inflammatory response. E. coli infection is also considered as an acute infectious disease. The relative increase in the range of lymphocyte and heterophil and relative decrease in the range of macrophage indicate mixed infection. The results are in agreement with a previous study (Villiers and Dunn, 1998). Evaluation of cellular responses can provide the veterinarian with a diagnostic aid in the development of a presumptive or definite diagnosis (Campbell et al., 1988). The present results provide a basis for the diagnosis and predilection of these diseases at post mortem. Although it is not confirmatory test, comparing with other diagnostic methods but advantageous to use the smear cytology due to its easiness as well as low expense and possible application for other histological test which is not possible on formalin fixed and paraffin embedded tissues. However, the cytological evaluation of Mycoplasma/E. coli infected birds has not been reported. When cytological diagnosis suggests Mycoplasma/E. coli, a more specific complementary examination should be added to confirm infection, so that adequate treatment can be done. This would probably reduce the treatment cost in poultry farming.

\section{CONCLUSION}

Cytological smear showed increased lymphocyte count $(\geq 85 \%)$ which can be considered as Mycoplasma infection and an increased heterophil count ( $\pm 19 \%)$ can be considered as E. coli infection. In case of Mycoplasma $-E$. coli complex, normal lymphocyte $\pm 78 \%$ count and $\pm 14 \%$ heterophil count can be considered. Cytological examination requires only glass slide and Giemsa stain; it could be a useful, rapid and compatible tool in field condition. Although it is not a confirmatory test, it could help pathologist to make decision one step further after post mortem examination that could guide a further confirmatory laboratory test. 


\section{REFERENCES}

Bottles, K., Miller, T. R., Cohen, M.B. and Ljung, B.M. 1986. Fine needle aspiration biopsy. Has its time come? Am. J. Med., 81: 525-531.

Campbell, T.W. 1988. Avian hematology and cytology. $1^{\text {st }}$ ed., Iowa State University Press, Ames.

Cochand-Priollet, B., Chagnon, S., Ferrand, J., Blery, M., Hoang, C. and Galian, A. 1987. Comparison of cytologic examination of smears and histologic examination of tissue cores obtained by fine needle aspiration biopsy of the liver. Acta. Ctyol., 31:476-480.

Cowell, R.L. and Tyler, R.D. 1989. Diagnostic cytology of the dog and cat. $1^{\text {st }}$ ed., American Veterinary Publications, California.

Chandrashekhar, C., Taryn, K., Gurpreet, A. and Baljit, S. 2008. Lung responses to secondary exotoxin challenge in rats exposed to pig barn air. J. Occup. Med. Toxicol., 3: 24.

Johnson, R.A. and Wichern, D.W. 2002. Applied Multivariate Statistical Analysis, Pearson Education, Inc., 5th edition, pp. 232-234.

Lauerman, L.H., Chilina, A.R., Closser, J.A. and Johansen, D. 1995. Avian Mycoplasma identification using Polymerase Chain Reaction Amplicon and Restriction Fragment Length Polymorphism Analysis. Avian Dis., 39: 804-811.

Luna, L. G. 1968. Manual of histologic staining methods of the Armed Forces Institute of Pathology. New York: McGraw-Hill Publications.200 pp.

Kanji, K.G. 1993. 100 statistical tests, SAGE Publications, pp. 86.

Nascimento, E.R., Pereira, V.L.A., Nascimento, M.G.F. and Barreto, M.L. 2005. Avian mycoplasmosis update. J. Vet. Med., 7: 1-9.

OIE (Office International des Epizooties/ World organization for Animal Health). 2008. Avian Mycoplasmosis. In Mannual of Diagnostic Tests and Vaccines for Terrestrial Animals. Chapter 2.3.5, pp: 482-496.

Sikder, A.J., Islam, M.A., Rahman, M.M. and Rahman, M.B. 2005. Seroprevalence of Salmonella and Mycoplasma gallisepticum infection in the six model breeder poultry Farms at Patuakhali district in Bangladesh. Cited from Asian Network for Scientific Information, pp 1682-8356.

Villiers, E. and Dunn. J. 1998. General Principal of cytological interpretation. Companion Animal Practice. pp. 429-437. 
Table 2: Inflammatory cell count in cytological smear from lungs obtained from normal healthy cases.

\begin{tabular}{cccc}
\hline Sample No & Lymphocyte (\%) & Heterophil (\%) & Macrophage (\%) \\
\hline N1 & 85 & 9 & 6 \\
N2 & 75 & 10 & 15 \\
N3 & 82 & 11 & 7 \\
N4 & 75 & 15 & 10 \\
N5 & 73 & 12 & 15 \\
N6 & 77 & 13 & 10 \\
N7 & 78 & 13 & 9 \\
N8 & 76 & 9 & 15 \\
N9 & 72 & 13 & 15 \\
N10 & 83 & 10 & 7 \\
\hline **Mean( \pm Margin & $77.6( \pm 4.06)$ & $11.5( \pm 1.87)$ & $10.9( \pm 3.48)$ \\
of error) & & & \\
\hline
\end{tabular}

Table 3: Inflammatory cell count in cytological smear from lungs obtained from Mycoplasma infected cases.

\begin{tabular}{cccc}
\hline Sample No & Lymphocyte (\%) & Heterophil (\%) & Macrophage (\%) \\
\hline M1 & 86 & 7 & 7 \\
M2 & 85 & 8 & 7 \\
M3 & 85 & 7 & 8 \\
M4 & 80 & 9 & 11 \\
M5 & 82 & 8 & 10 \\
M6 & 90 & 6 & 3 \\
M7 & 88 & 6 & 6 \\
M8 & 86 & 6 & 8 \\
M9 & 84 & 5 & 11 \\
\hline **Mean( \pm Margin & $85.11( \pm 2.99)$ & $6.88( \pm 1.28)$ & $7.1( \pm 2.58)$ \\
of error) & & & \\
\hline
\end{tabular}


Table 4: Inflammatory cell count in cytological smear from lungs obtained from $E$. coli infected cases

\begin{tabular}{cccc}
\hline Sample No & Lymphocyte (\%) & Heterophil (\%) & Macrophage (\%) \\
\hline E1 & 69 & 19 & 12 \\
E2 & 69 & 20 & 11 \\
E3 & 71 & 19 & 10 \\
E4 & 61 & 24 & 15 \\
E5 & 70 & 21 & 9 \\
E6 & 70 & 22 & 8 \\
E7 & 69 & 21 & 10 \\
E8 & 68 & 22 & 10 \\
E9 & 67 & 23 & 10 \\
E10 & 62 & 25 & 13 \\
\hline **Mean( \pm Margin & $67.6( \pm 3.16)$ & $21.6( \pm 1.87)$ & $10.8( \pm 1.90)$ \\
of error & & & \\
\hline
\end{tabular}

Table 5: Inflammatory cell count in cytological smear from lungs obtained from mixed infected cases.

\begin{tabular}{cccc}
\hline Sample No & Lymphocyte (\%) & Heterophil (\%) & Macrophage (\%) \\
\hline M+E1 & 75 & 17 & 8 \\
M+E2 & 75 & 20 & 5 \\
M+E3 & 78 & 15 & 7 \\
M+E4 & 78 & 16 & 6 \\
M+E5 & 80 & 13 & 7 \\
M+E6 & 81 & 14 & 5 \\
M+E7 & 78 & 15 & 7 \\
M+E8 & 79 & 14 & 7 \\
M+E9 & 81 & 16 & 3 \\
\hline **Mean( \pm Margin & $78.33( \pm 2.25)$ & $15.55( \pm 2.08)$ & $6.11( \pm 1.55)$ \\
of error) & & & \\
& & & \\
\hline
\end{tabular}


Table 6: The average percentage of inflammatory cells in cytological smear from lungs obtained from normal healthy, Mycoplasma, E. coli and mixed infection cases.

\begin{tabular}{|c|c|c|c|}
\hline \multirow{2}{*}{ Groups } & \multicolumn{3}{|c|}{ The average cell percentage Mean ( \pm SD) } \\
\cline { 2 - 4 } & Lymphocyte (\%) & Heterophil (\%) & Macrophage (\%) \\
\hline $\begin{array}{c}\text { Normal healthy } \\
\text { (control) }\end{array}$ & $77.6 \pm 4.06$ & $11.5 \pm 1.87$ & $10.9 \pm 3.48$ \\
\hline $\begin{array}{c}\text { Mycoplasma } \\
\text { infected }\end{array}$ & $85.11 \pm 2.99$ & $6.88 \pm 1.28$ & $7.1 \pm 2.58$ \\
\hline E.coli infected & $67.6 \pm 3.16$ & $21.6 \pm 1.87$ & $10.8 \pm 1.90$ \\
\hline Mixed infected & $78.33 \pm 2.25$ & $15.55 \pm 2.08$ & $6.11 \pm 1.55$ \\
\hline
\end{tabular}

** Margin of Error $=t_{n-1}\left(\frac{\alpha}{2 p}\right) \frac{S D_{i i}}{\sqrt{n}}$ where $t_{n-1}\left(\frac{\alpha}{2 p}\right)$ Critical Values for Bonferroni's method of multipe comparisons, $S D$ refers to standard deviation, $n$ refers to sample size.
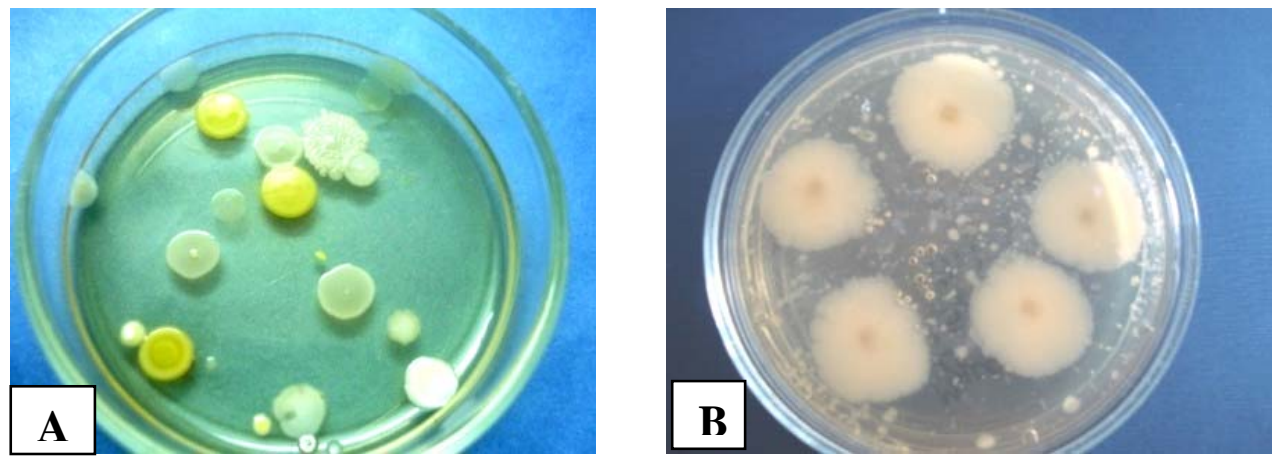

Figure 1. Growth of Mycoplasma supplemented with horse serum. A-B: Fried egg appearance with formation of nipple at the center, A: 8 days culture, B: 18 days culture. 


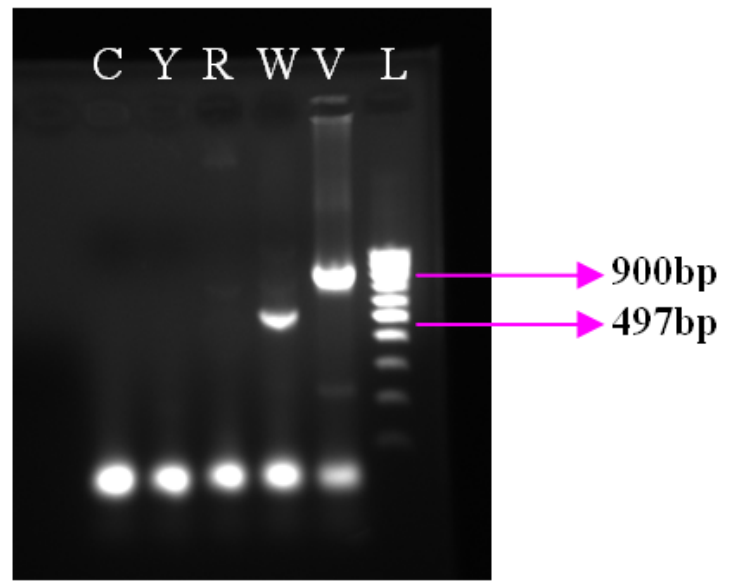

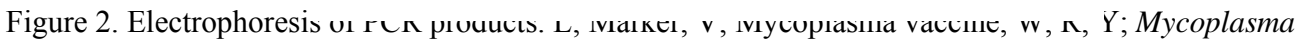
samples, C; Water control. V; Vaccine (M. gallisepticum) and W; M. synoviae showed positive band $900 \mathrm{bp}$ and $497 \mathrm{bp}$, respectively.

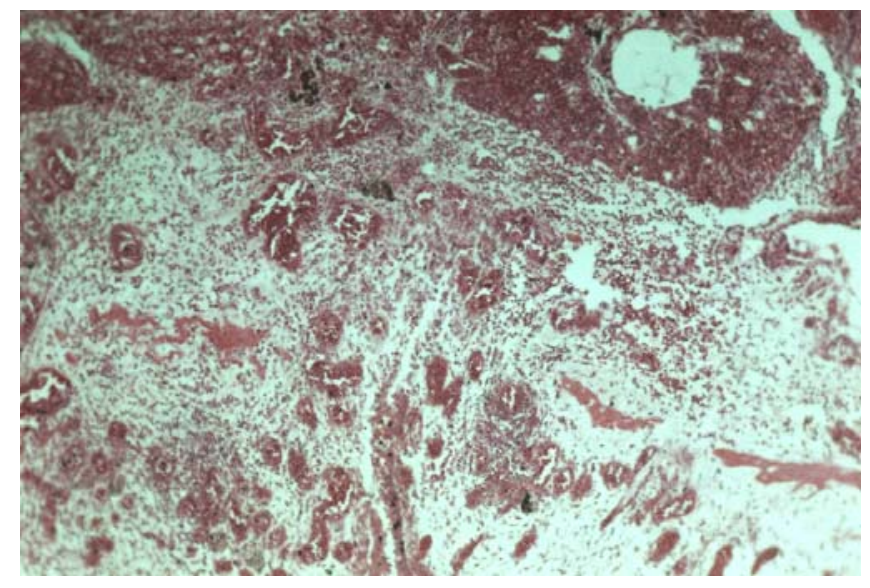

Figure 3. Pneumonia with predominant lymphocytic infiltration (H\&E stain x 330). 


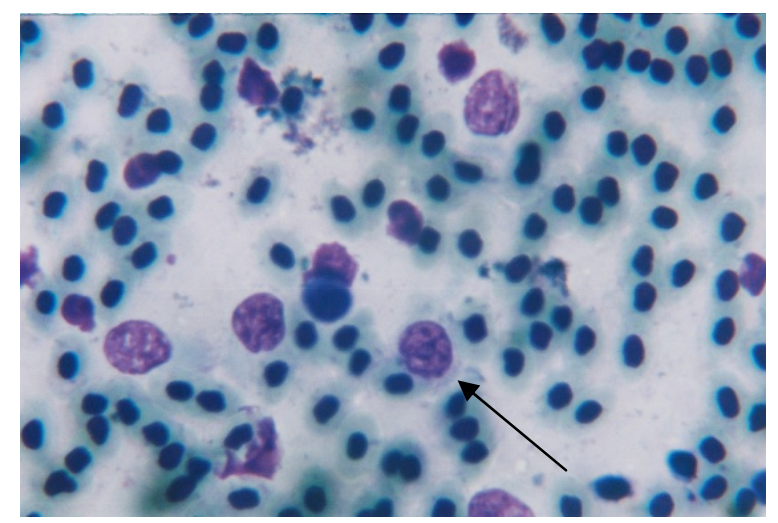

Figure 4. Lung smear from Mycoplasma infected bird consisting mainly lymphocyte (arrow). Giemsa stain x 825 .

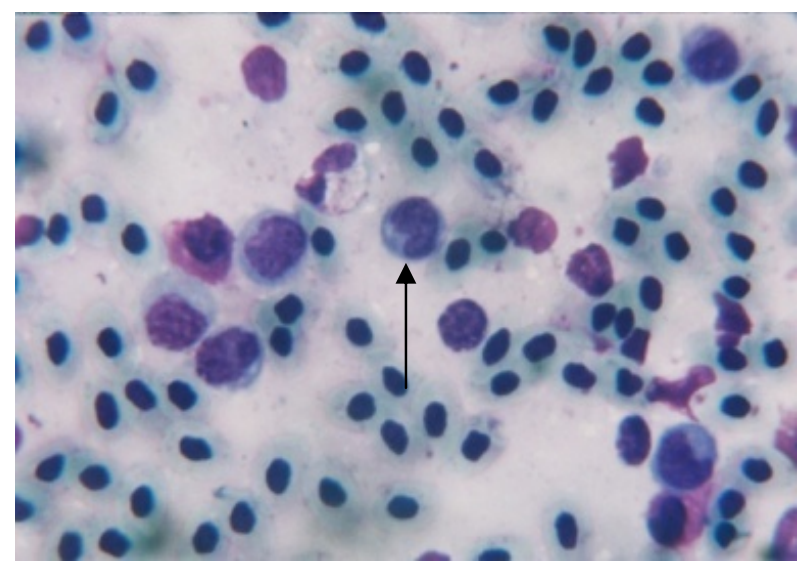

Figure 5. Lung smear from Mycoplasma infected cases consisting mainly macrophage (arrow), Giemsa stain, $\times 82.5)$.

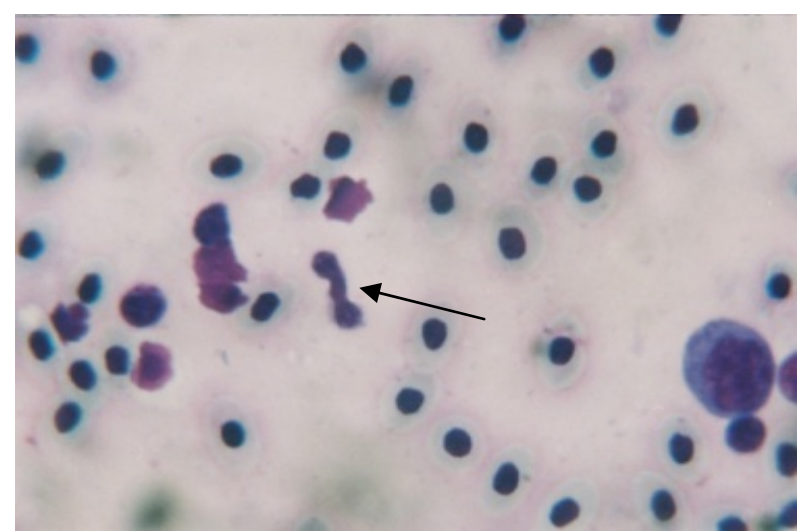

Figure 6. Lung smear from E.coli infected cases consisting mainly heterophil (arrow), Giemsa stain, $\times 82.5$ ). 